\title{
Theoretical calculation for the ultraviolet optical properties of single-walled carbon nanotubes
}

\author{
Yoshiteru Takagi and Susumu Okada \\ Center for Computational Sciences and Graduate School of Pure and Applied Sciences, University of Tsukuba, Tsukuba 305-8571, Japan \\ and CREST, Japan Science and Technology Agency, 4-1-8 Honcho, Kawaguchi, Saitama 332-0012, Japan
}

(Received 25 March 2009; revised manuscript received 19 May 2009; published 19 June 2009)

\begin{abstract}
We present a theoretical study of the ultraviolet (UV) optical properties of carbon nanotubes based on the local-density approximation in density functional theory and the generalized tight-binding approximation. Our calculations indicate that the absorption spectrum in the UV region depends strongly not only on nanotube diameter but also on chiral index. In zigzag nanotubes with even indices, peculiar features in the UV absorption spectrum emerge. In sharp contrast, these features are absent from the spectrum of zigzag nanotubes with odd indices. These spectral features are characteristic of interband transitions near the $M$ point of the hexagonal Brillouin zone of graphene.
\end{abstract}

DOI: $10.1103 /$ PhysRevB.79.233406

PACS number(s): 78.40.Ri, 71.20.Tx, 78.20.Bh

Carbon nanotubes ${ }^{1}$ have attracted significant attention from the pure and applied sciences due to their unique structural and electronic properties. ${ }^{2}$ One of the most fascinating aspects of nanotubes is that they exhibit either metallic or semiconducting electronic properties depending on their diameter and the atomic arrangement along their circumference. ${ }^{3,4}$ The unusual electronic properties of nanotubes open the possibility of fabricating superior nanometerscale electronic devices in which the nanotubes would be a key constituent. For instance, it has been demonstrated that individual semiconducting nanotubes can work as field-effect transistors with properties that depend strongly on the nanotube species and the metal electrode species. ${ }^{5-7}$ Although knowledge of nanotube devices is steadily accumulating, difficulties in characterizing and extracting individual nanotubes slows the progress toward industrial applications.

For various applications, the use of nanotubes requires the characterization of the diameter and the chiral angle of each individual single-walled carbon nanotube. Optical spectroscopic techniques are one promising avenue for identifying and probing the geometric structure of nanotubes. The electronic structure of a nanotube, which is obtained by applying the discretization of the energy band of graphene, is directly affected by its atomic arrangement and diameter. Thus, careful investigation of the optical spectrum gives the geometric structure of individual nanotubes. Indeed, by comparing absorption spectra with electronic structures theoretically obtained, it is possible to identify not only the diameter but also the chiral index of each nanotube. The sensitive dependence of the low-energy (i.e., visible and near infrared) absorption spectrum on tube diameter has been experimentally demonstrated by Kataura et al. ${ }^{8}$ More recently, detailed ultraviolet (UV) spectroscopic experiments also clearly indicate that the spectrum depends strongly on tube diameter. ${ }^{9,10}$ However, to our knowledge, ${ }^{11,12}$ the calculation of the UV absorption spectrum is not yet addressed. Furthermore, the origin of the features in the UV spectra are still unclear. ${ }^{8,13-17}$

The purpose of this Brief Report is therefore to explain theoretically the optical properties of nanotubes in UV region. To quantitatively discuss interband UV transitions we study the absorption coefficient of nanotubes in which the eigenstates are evaluated using the first-principle total-energy calculation in the framework of density functional theory
(DFT). We also find more peaks in the absorption spectrum for zigzag nanotubes with even indices compared with that for zigzag nanotubes with odd indices [see Figs. 1(a) and 1(b)]. To complement the DFT results we also investigate the absorption coefficient for nanotubes with diameters less than

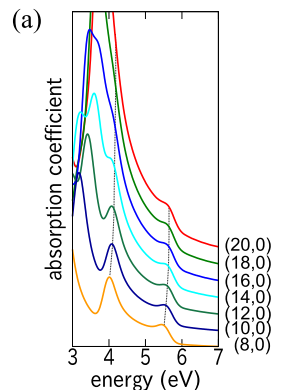

(c)

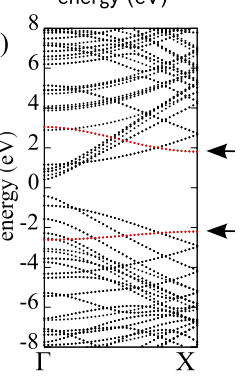

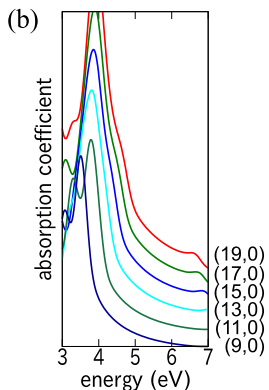

(d)

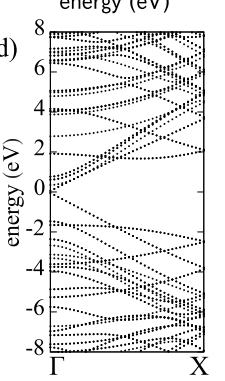

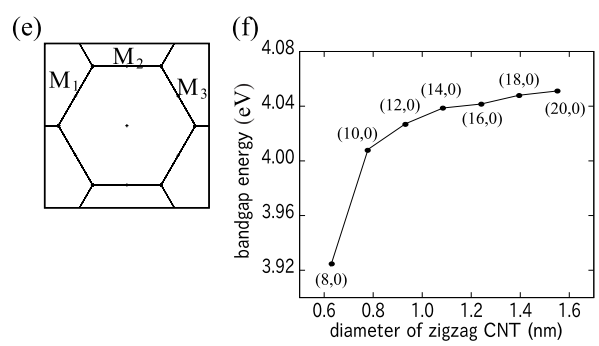

FIG. 1. (Color online) Absorption coefficients $(\alpha)$ in UV region for zigzag nanotubes (a) with even indices and (b) with odd indices calculated by using the LDA. Indices of nanotubes are listed in the right margin of each figure. Electronic structures of (c) the $(10,0)$ nanotube and (d) the $(9,0)$ nanotube. (e) Two-dimensional Brillouin zone with the hexagonal symmetry. $M_{1}, M_{2}$, and $M_{3}$ indicate independent $M$ point in the Brillouin zone. (f) Energy gap of zigzag nanotube with even indices at $X$ point on their diameter. 
14.0 A. Our calculations indicate that peak shifts in the UV absorption spectrum also depends strongly on nanotube diameter as is the case for the low-energy absorption spectrum. The calculated shifts in the spectral peaks agree to an excellent degree with those observed experimentally. The results of the calculation indicate that the interband transition significantly affects the UV absorption spectrum of nanotubes.

To obtain one-electron states of individual nanotubes, we perform first-principle calculations using the local-density approximation (LDA) within the density functional theory. ${ }^{18,19}$ For the exchange-correlation energy between electrons, we use a functional ${ }^{20}$ fitted to the Monte Carlo results for a homogeneous electron gas. ${ }^{21}$ Norm-conserving pseudopotentials generated using the Troullier-Martins scheme are adopted to describe the electron-ion interaction. $^{22,23}$ The valence wave functions are expanded using the plane-wave basis set with a cutoff energy of $49 \mathrm{Ry}$, making the relative total energies of carbon-related materials sufficiently convergent. ${ }^{22,24}$ We adopt a supercell model in which a nanotube is situated with its wall separated from the walls of adjacent nanotubes by about $7.0 \AA$. The conjugategradient minimization scheme is utilized both for the electronic-structure calculation and for the geometry optimization. $^{25}$ The lattice-parameter $c$ is fixed during the structural optimization. In the optimized geometry, atoms are subjected to a force of less than 0.001 Hartree/a.u. integration over the one-dimensional Brillouin zone is carried out using the eight $k$ points. The actual calculations were carried out using the Tokyo ab initio program package (TAPP). ${ }^{26,27}$

In addition to the LDA in DFT we also use the tightbinding approximation to obtain the comprehensive electronic structure of the nanotubes. Here, $2 s$ and $2 p$ states of the carbon atom are used as the basis set to discuss the $\pi-\sigma$ rehybridization induced by the nanotube curvature. Transfer and overlap integrals between neighboring atomic orbitals are suitably distance dependent in order to reproduce the electronic band structure for not only occupied states but also unoccupied states of various atomic configurations of the carbon atom. ${ }^{28}$ Furthermore, the nonorthogonality of the atomic orbitals on neighboring sites is fully taken into account. We set the cutoff radius to $4.0 \AA$ in the tight-binding parameter set.

The absorption coefficient of the nanotubes, $\alpha(\omega)$, is evaluated by calculating

$$
\alpha(\omega)=\frac{4 \pi^{2}}{\omega V} \sum_{c, \mathbf{k}^{\prime}} \sum_{v, \mathbf{k}}\left|\left\langle\mathbf{k}^{\prime}, c|\mathbf{e} \cdot \mathbf{p}| \mathbf{k}, v\right\rangle\right|^{2} \delta\left[\boldsymbol{\epsilon}_{c}\left(\boldsymbol{k}^{\prime}\right)-\boldsymbol{\epsilon}_{v}(\boldsymbol{k})-\omega\right],
$$

where $\mathbf{e}$ is the light polarization vector, $\mathbf{p}$ is the electron momentum, $V$ is the unit cell volume, $\omega$ is the photon energy, $\epsilon_{c}(\mathbf{k})$ and $\epsilon_{v}(\mathbf{k})$ are one-electron energies at momentum $\mathbf{k}$ for valence and conduction band electrons, respectively, and $|\mathbf{k}, c\rangle$ and $|\mathbf{k}, v\rangle$ are eigenstates in the valence and conduction band, respectively. In this work, we focus on optical interband transitions with the light-polarization vector parallel to the tube axis.

Figures 1(a) and 1(b) show the optical absorption spectra calculated using the LDA in the DFT of zigzag nanotubes possessing even and odd indices, respectively. We find clear features at about 4 and $5.5 \mathrm{eV}$ in the absorption spectrum of zigzag nanotubes with even indices [Fig. 1(a)]. These features are found only for the even-index nanotubes and are absent from the absorption spectrum of zigzag nanotubes with odd indices [Fig. 1(b)]. We expect that the differences in the absorption spectra of nanotubes with even or odd indices reflect the electronic structure of individual nanotubes since absorption spectra are determined by energy-band dispersion.

To elucidate this issue, we focus on the electronic structure of zigzag nanotubes. In Figs. 1(c) and 1(d) we show the electronic structure of zigzag nanotubes with chiral indices $(10,0)$ and $(9,0)$, which are chosen as representative of zigzag nanotubes with even and odd chiral indices. By comparing these two electronic structures, we find a substantial difference at $2 \mathrm{eV}$ above and below the Fermi level, where a pair of relatively flat dispersion bands [indicated by arrows in Fig. 1(c)] appear in the electronic structure of the $(10,0)$ nanotube. No such state is present for the $(9,0)$ nanotube [Fig. 1(d)]. The flat dispersion bands arise from the energy band of $\pi$ and $\pi^{*}$ electrons along the two adjacent $M$ points, $M_{2}$ and $M_{3}$, in the two-dimensional Brillouin zone of graphene [see Fig. 1(e)].

Analysis of the $(10,0)$ nanotube band structure reveals the origin of the characteristic two-peak structure of the absorption spectrum of zigzag nanotubes with even indices, which comes from the interband transition between the $\pi$ and $\pi^{*}$ bands at the $X$ and $\Gamma$ points, where they have relatively flat dispersion, resulting in the high- and low-energy features, respectively. Thus, the UV absorption spectrum exhibits spectral features that are characteristic of nanotubes with odd or even chiral indices.

In addition to the features in the UV absorption spectrum depending on chirality, we also find that they are sensitive to nanotube diameter. As clearly shown in Fig. 1(a), the absorption peaks near 4.0 and $5.5 \mathrm{eV}$ shift to higher energy with increasing nanotube diameter. The blueshift reflects the modulation of energy gap between the $\pi$ and $\pi^{*}$ bands, both of which exhibit relatively flat dispersion at the $X$ and at $\Gamma$ points: a decrease in curvature results in an upward shift for the $\pi^{*}$ band and a downward shift for the $\pi$ band. ${ }^{29}$ The calculated energy gap at the $X$ point is shown in Fig. 1(f) as a function of nanotube diameter. The gap increases with increasing the tube diameter supporting the suggestion that the characteristic absorption features result from $\pi-\pi^{*}$ transitions. Indeed, the gap at $X$ point of nanotubes excellently agrees with the adsorption spectra peak at around $4 \mathrm{eV}$ indicating the fact that the curvature effects are totally ascribed to the interband transition.

Our DFT calculations for zigzag nanotubes indicate that the interband transition is responsible for the significant features in the UV absorption spectrum. To complement the results obtained by first-principle calculations, we use tightbinding calculations to study the absorption spectrum of carbon nanotubes of with diameters less than $14 \AA$. Figure 2 shows the absorption coefficient as a function of energy calculated using the tight-binding approximation for $(10, m)$ and $(9, m)$ nanotubes. We find that the UV spectral features allow the separation of the spectra into four classes. The first class contains the $(10, m)$ nanotubes with even index $m$, which 
(a)

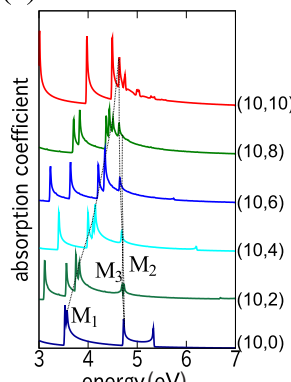

(c)

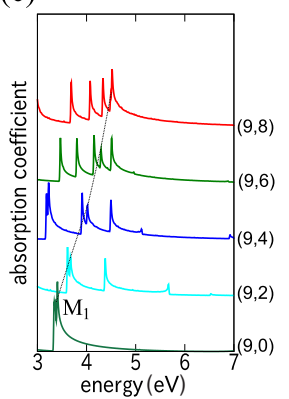

(b)

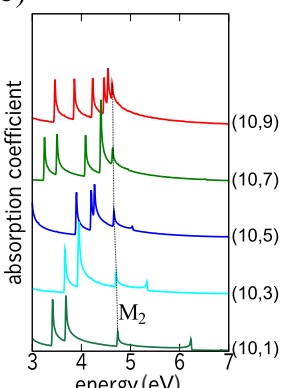

(d)

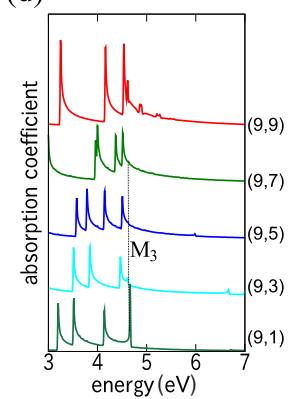

FIG. 2. (Color online) Absorption coefficients $(\alpha)$ in UV region for $(10, m)$ nanotubes with (a) even indices $m$ and (b) odd indices $m$ calculated by the tight-binding approximation. Absorption coefficients in UV region for $(9, m)$ nanotubes with (c) even indices $m$ and (d) odd indices $m$. Broken lines denote guide for eye to identify the peak position of spectra attributed from $M$ points.

display three distinct features of which one depends strongly on the chirality. In this class, the peak in the absorption coefficient shifts from 3.5 to $4.5 \mathrm{eV}$ with increasing index $m$ or chiral angle. In sharp contrast, the remaining two features, which are almost degenerate at approximately $4.7 \mathrm{eV}$, are insensitive to chiral angle. The other three classes consist of the $(10, m)$ nanotubes with odd index $m$ and $(9, m)$ nanotubes with either odd or even indices. These classes display only one absorption feature in the UV region attributed to an $M$ point. Among these three latter classes of nanotubes, a significant dependence on chiral angle occurs only in $(9, m)$ nanotubes with even index $m$ where the peak also shifts from 3.5 to $4.5 \mathrm{eV}$ with increasing chiral angle.

The characteristics of the UV spectra are attributed to wave vectors that contain the $M$ point in the two-dimensional hexagonal Brillouin zone of graphene. In the case of nanotubes whose indices are all even, the three UV features arise from the three independent $M$ points allowed on the discretized wave vector of the Brillouin zone of graphene. On the other hand, the remaining three nanotubes classes involve only one of the three $M$ points. The wave vector of nanotubes with odd-even index, even-odd index, and odd-odd index contains the points $M_{1}, M_{2}$, and $M_{3}$ of the Brillouin zone of graphene, respectively. Among these three distinct $M$ points, the UV absorption feature ascribed to $M_{1}$ point exhibits a substantial dependence on chiral angle. Indeed, the peak shift from 3.5 to $4.5 \mathrm{eV}$ discussed above is only observed in $(n, m)$ nanotubes with even index $m$. We note that a dipole moment between the $\pi$ and $\pi^{*}$ electrons at the $M_{3}$ point vanishes in the armchair nanotubes. Furthermore, by increasing the chiral angle the three distinct features in the

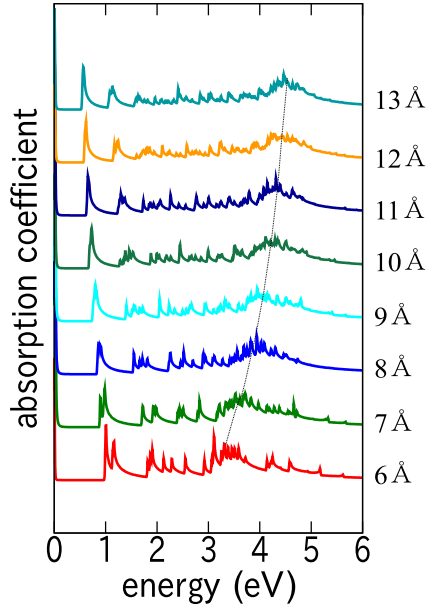

FIG. 3. (Color online) Averaged absorption spectra of nanotubes for various diameter ranges. Representative diameters for average spectra are listed in the right margin of the figure. Broken lines denote guide for eye to identify the peak shift in spectra.

UV absorption spectrum approach $4.7 \mathrm{eV}$, which corresponds to the energy gap between $\pi$ and $\pi^{*}$ states at the $M$ point of graphene.

Experimentally observed absorption spectra, however, are averaged over a number of nanotubes with varying chirality. Thus, to compare theoretical and experimental spectra, we take a simple sum of the spectra of all nanotubes with diameter $d$ from $d$ to $d+1 \AA$. Figure 3 shows the averaged absorption spectra for nanotubes with $d=6 \AA \sim 13 \AA$ calculated by using the tight-binding approximation. We find that features in UV region tend to shift to higher energy with increasing the tube diameter. This trend qualitatively agrees with that observed experimentally in absorption spectra. ${ }^{10}$ Thus we conclude that the dependence of the experimental UV absorption spectra on nanotube diameter is at least partly caused by the interband transition at and around the $M$ point of graphene.

In summary, using both first-principle and tight-binding calculations, we study the UV optical properties of carbon nanotubes. For zigzag nanotubes with even indices, our firstprinciple calculations show two substantial features in UV region, whereas these features are absent from the absorption spectrum of zigzag nanotubes with odd indices. These features arise from interband transitions between the relatively low-dispersion bands discretized from the $M-M$ lines in the hexagonal Brillouin zone of graphene. In addition, the features are found to shift to higher energy with increasing tube diameter. Tight-binding calculations also indicate that the peaks in the absorption spectrum originate from interband transitions near the $M$ point. Moreover, we find a dependence of the UV absorption spectrum on the parity of the chiral index. We expect that combining our results with the low-energy spectral signatures of the $3 n \pm 1$ family will lead to a more accurate characterization of nanotubes.

We thank K. Yanagi and T. Saito for communicating their results prior to publication. This work was partly supported by CREST in Japan Science and Technology Agency and a 
grant-in-aid for scientific research from the Ministry of Education, Culture, Sports, Science and Technology of Japan. Computations were performed on NEC SX series at the University of Tsukuba, at the Yukawa Institute of Theoretical
Physics, Kyoto University, at the Cybermedia Center, Osaka University, at the Information Synergy Center, Tohoku University, and at the Research Center of Computational Science, Okazaki National Institute.
${ }^{1}$ S. Iijima, Nature (London) 354, 56 (1991).

${ }^{2}$ M. S. Dresselhaus G. Dresselhaus, and P. C. Eklund, Science of Fullerenes and Carbon Nanotubes (Academic Press, San Diego, 1996).

${ }^{3}$ N. Hamada, S. I. Sawada, and A. Oshiyama, Phys. Rev. Lett. 68, 1579 (1992).

${ }^{4}$ R. Saito, M. Fujita, M. S. Dresselhaus, and G. Dresselhaus, Appl. Phys. Lett. 60, 2204 (1992).

${ }^{5}$ S. J. Tans, A. R. M. Verschueren, and C. Dekker, Nature (London) 393, 49 (1998).

${ }^{6}$ R. Martel, T. Schmidt, H. R. Shea, T. Hartel, and Ph. Avouris, Appl. Phys. Lett. 73, 2447 (1998).

${ }^{7}$ Y. Nosho, Y. Ohno, S. Kishimoto, and T. Mizutani, Appl. Phys. Lett. 86, 073105 (2005).

${ }^{8}$ H. Kataura, Y. Kumazawa, Y. Maniwa, I. Umezu, S. Suzuki, Y. Ohtsuka, and Y. Achiba, Synth. Met. 103, 2555 (1999).

${ }^{9}$ T. Saito, S. Ohshima, T. Okazaki, S. Ohmori, M. Yumura, and S. Iijima, J. Nanosci. Nanotechnol. 8, 6153 (2008).

${ }^{10} \mathrm{~K}$. Yanagi and T. Saito (private communication).

${ }^{11}$ M. F. Lin and Kenneth W.-K. Shung, Phys. Rev. B 50, 17744 (1994).

${ }^{12}$ G. Y. Guo, K. C. Chu, D.-S. Wang, and C.-G. Duan, Phys. Rev. B 69, 205416 (2004).

${ }^{13}$ T. Pichler, M. Knupfer, M. S. Golden, J. Fink, A. Rinzler, and R. E. Smalley, Phys. Rev. Lett. 80, 4729 (1998).

${ }^{14}$ B. W. Reed and M. Sarikaya, Phys. Rev. B 64, 195404 (2001).

${ }^{15}$ Y. Murakami, E. Einarsson, T. Edamura, and S. Maruyama, Phys. Rev. Lett. 94, 087402 (2005).
${ }^{16}$ N. E. Itkis, D. E. Perea, R. Jung, S. Niyog, and R. C. Haddon, J. Am. Chem. Soc. 127, 3439 (2005).

${ }^{17}$ C. Kramberger, R. Hambach, C. Giorgetti, M. H. Rümmeli, M. Knupfer, J. Fink, B. Büchner, Lucia Reining, E. Einarsson, S. Maruyama, F. Sottile, K. Hannewald, V. Olevano, A. G. Marinopoulos, and T. Pichler, Phys. Rev. Lett. 100, 196803 (2008).

${ }^{18}$ P. Hohenberg and W. Kohn, Phys. Rev. 136, B864 (1964).

${ }^{19}$ W. Kohn and L. J. Sham, Phys. Rev. 140, A1133 (1965).

${ }^{20}$ J. P. Perdew and A. Zunger, Phys. Rev. B 23, 5048 (1981).

${ }^{21}$ D. M. Ceperley and B. J. Alder, Phys. Rev. Lett. 45, 566 (1980).

${ }^{22}$ N. Troullier and J. L. Martins, Phys. Rev. B 43, 1993 (1991).

${ }^{23}$ L. Kleinman and D. M. Bylander, Phys. Rev. Lett. 48, 1425 (1982).

${ }^{24}$ S. Okada, S. Saito, and A. Oshiyama, Phys. Rev. Lett. 86, 3835 (2001).

${ }^{25}$ O. Sugino and A. Oshiyama, Phys. Rev. Lett. 68, 1858 (1992).

${ }^{26} \mathrm{M}$. Tsukada, Computer program package TAPP, University of Tokyo, Tokyo, Japan, 1983-2009.

${ }^{27}$ J. Yamauchi, M. Tsukada, S. Watanabe, and O. Sugino, Phys. Rev. B 54, 5586 (1996).

${ }^{28}$ D. Papaconstantopoulos, M. Mehl, S. Erwin, and M. Pederson, Tight-Binding Approach to Computational Materials Science, edited by E. A. Turchi, A. Gonis, and L. Columbo, MRS Symposia Proceedings No. 491 (Material Research Society, Warrendale, PA, 1998), pp. 221.

${ }^{29}$ S. Reich, C. Thomsen, and P. Ordejón, Phys. Rev. B 65, 155411 (2002). 